\title{
Pilomatricoma recidivado em região superciliar esquerda: relato de caso
}

\author{
Recurrent pilomatricoma on the left eyebrow: case report
}

\author{
Marcio Henrique Mendes ${ }^{1}$ \\ Leoni de Paiva Souza ${ }^{2}$
}

Trabalho realizado na Santa Casa de Santo Amaro - São Paulo - (SP) - Brasil.

${ }^{1}$ Estagiário do Setor de Ecografia do Hospital das Clínicas da Faculdade de Medicina da Universidade de São Paulo - USP - São Paulo (SP) - Brasil.

${ }^{2}$ Estagiário do Setor de Vias Lacrimais e Estrabismo da Faculdade de Medicina da Universidade do ABC Santo André (SP) - Brasil.

Endereço para correspondência: Marcio Henrique Mendes. Rua Tabapuã, 474 - $8^{\circ}$ andar - São Paulo (SP) CEP 04533-001

E-mail: marciohmendes@yahoo.com.br

Recebido para publicação em 01.11.2006

Última versão recebida em 14.12.2008

Aprovação em 14.01.2009

Nota Editorial: Depois de concluída a análise do artigo sob sigilo editorial e com a anuência da Dra. Eliana Aparecida Forno sobre a divulgação de seu nome como revisora, agradecemos sua participação neste processo.

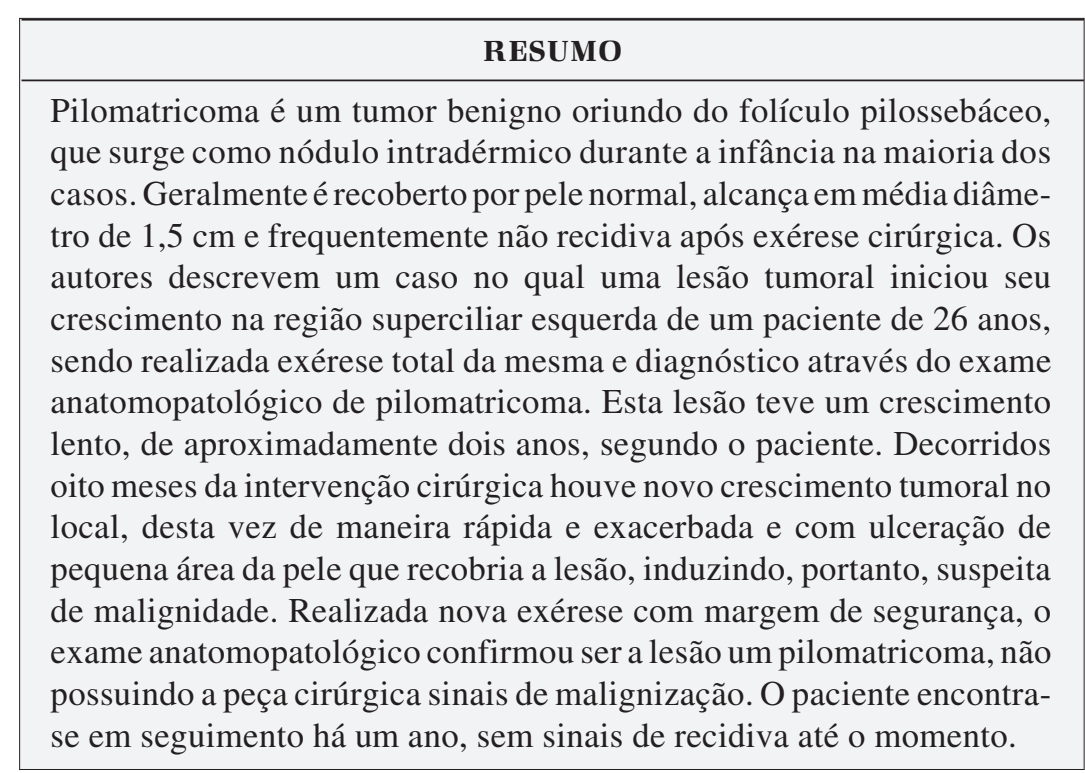

Descritores: Pilomatricoma/cirurgia; Recidiva; Neoplasias cutâneas; Relatos de casos; Humanos; Masculino; Adulto [Tipo de publicação]

\section{INTRODUÇÃ̃O}

O pilomatricoma é um tumor cutâneo benigno, descrito originalmente por Malherbe \& Chenantais como uma lesão originária de glândulas sebáceas, denominando estas lesões na ocasião como epitelioma calcificante de Malherbe. Decorridos 81 anos desta primeira descrição, foi proposta nova denominação para este tipo de lesão por Forbis \& Helwig, que após estudarem 228 casos, concluíram que o tumor era originado da matriz pilosa, o denominando então como pilomatricoma, que por motivos etimológicos posteriormente passou a ser chamado de pilomatricoma ${ }^{(1-3)}$.

Surge em geral na infância, como um único nódulo intra-dérmico móvel de consistência pétrea, frequentemente recoberto por pele normal ${ }^{(4)}$, no entanto, podendo apresentar coloração rósea, avermelhada ou azulada. Excepcionalmente evidencia-se ulcerações. Situado principalmente na face, pescoço e membro superiores, embora haja relatos de incidência no tórax, abdome e membros inferiores. Alcança em média $1,5 \mathrm{~cm}$ de diâmetro ${ }^{(5)}$, havendo relatos de formas maiores, com até $13,5 \mathrm{~cm}^{(1)}$. Tem predileção pela raça branca e sexo feminino. Em $40 \%$ dos casos o início se dá na primeira década de vida, $60 \%$ antes dos vinte anos e apenas $13 \%$ após os cinquenta $\operatorname{anos}^{(6)}$. O tratamento consiste na excisão da lesão, não havendo recidiva como regra. 
É considerado um tumor raro. Moehlenbeck encontrou um pilomatricoma em cada 824 espécimes de biópsia e há outro estudo demonstrando 1:2.200 espécimes. Alguns autores, no entanto, acham que não seja tão raro e sim mal diagnosticado ${ }^{(7)}$.

\section{RELATO DE CASO}

Paciente branco do sexo masculino, 26 anos, com queixa de lesão tumoral em região superciliar esquerda pouco mais de seis meses antes do exame oftalmológico. Relatou ter sido submetido à exérese da mesma lesão um ano e quatro meses antes, tendo este tumor evoluído durante três anos, sem alterações da textura ou coloração da pele. O paciente possuía laudo de exame anatomopatológico prévio, com diagnóstico de pilomatricoma, medindo na ocasião $1,5 \times 1,5 \times 1,0 \mathrm{~cm}$.

Desta vez a lesão apresentou crescimento mais rápido. A pele que recobria a mesma apresentava ulceração com $1 \mathrm{~cm}$ de diâmetro, coloração avermelhada, consistência endurecida, sendo possível mobilizá-la, sugerindo não adesão a planos profundos (Figura 1).

O paciente foi submetido a exérese sob anestesia local e a reconstituição se deu por aproximação direta das bordas de pele não acometida com mononylon 6.0 (Figura 2). O tumor apresentava 4,5 x 2,5 x 1,5 cm, havendo lobulações (facetas) e pontos esbranquiçados e endurecidos, sugerindo calcificações por toda sua extensão (Figura 3). O exame anatomopatológico confirmou o diagnóstico anterior, não evidenciando sinais de malignidade.

O paciente encontra-se em seguimento há um ano sem sinal de recidiva.



Figura 1 - Lesão tumoral em região superciliar esquerda, recoberta por pele avermelhada e com ulceração central de $1 \mathrm{~cm}$ de diâmetro

\section{DISCUSS ÃO}

Esse tipo de tumor apresenta evolução lenta e progressiva, havendo, no entanto, casos de crescimento rápido. Pode apresentar aspectos incomuns, sendo descritas formas verrucosas, xantomatosas, ulceradas e pseudo-bolhosas ${ }^{(8)}$. A consistência também pode variar de pétrea à elástica, sendo a segunda mais rara. Nestes casos com consistência elástica se torna difícil a diferenciação com cisto epidérmico e dermatofibroma.

O diagnóstico clínico é considerado quase impossível, ao passo que deve ser diferenciado entre várias lesões clinica-

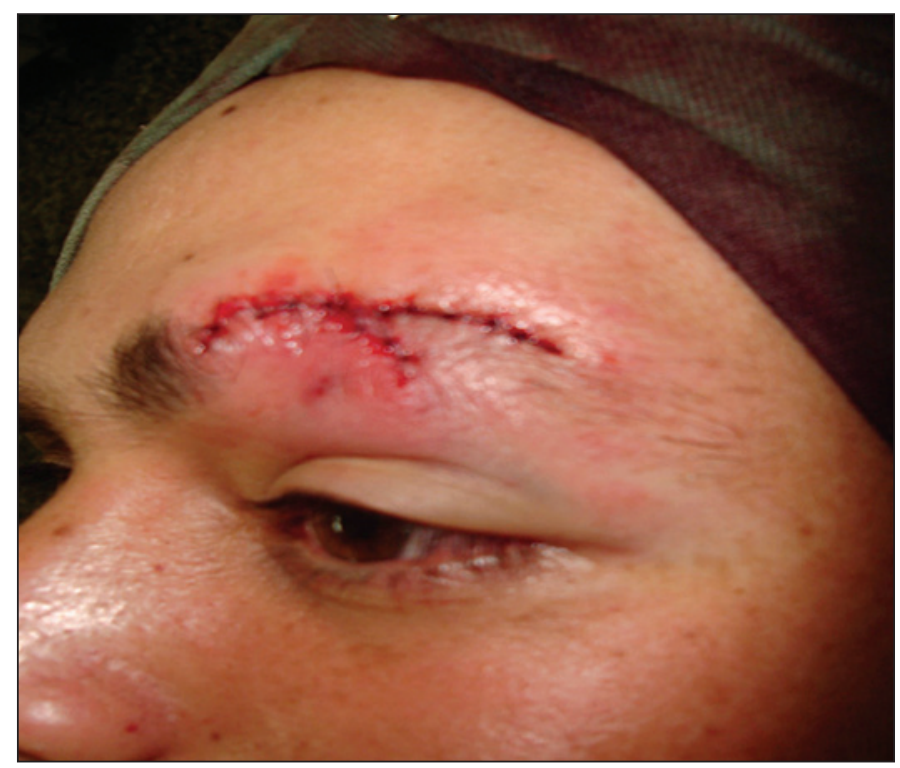

Figura 2 - Região superciliar apresentando bordas excisionais suturadas logo após exérese da lesão

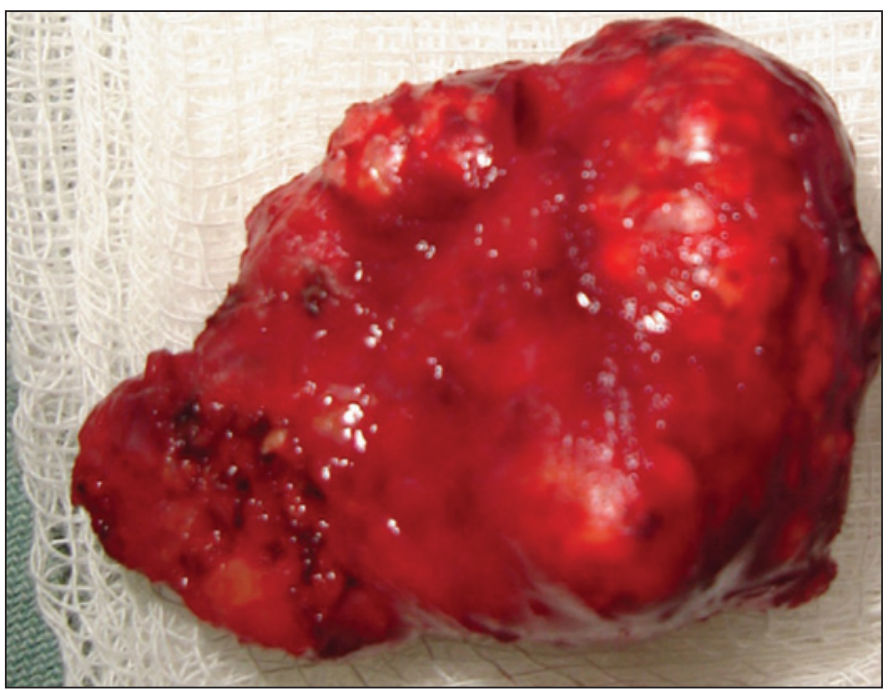

Figura 3 - Lesão tumoral medindo $4,5 \times 2,5 \times 1,5 \mathrm{~cm}$, de coloração avermelhada multilobulada, recoberta por membrana translúcida, apresentando pontos brancos por quase toda sua extensão, os quais indicam calcificações 
mente semelhantes, como: hemangioma, dermatofibroma, carcinomas baso e espinocelular, goma bacteriana ulcerada, angiossarcoma, melanoma e calcificações cutâneas, dentre outras.

Histopatologicamente observa-se localização na derme profunda ou no tecido celular subcutâneo, sendo característica a presença de massas tumorais arredondadas, bem demarcadas, circundadas por cápsula conjuntival (facetas). As ilhotas tumorais apresentam células basofílicas e células-sombra. Em $75 \%$ a $84 \%$ dos tumores podem ser observados depósitos de cálcio, que se apresentam como grânulos basofílicos no interior do citoplasma das células-sombra ou, ainda, como grandes massas amorfas intensamente basofílicas substituindo estas células-sombra. Ocasionalmente ocorrem depósitos estromais de cálcio ${ }^{(9-12)}$. No presente caso observam-se tais características (Figura 4).

É importante salientar a existência da forma "perfurante" do pilomatricoma, na qual ocorre eliminação transepitelial de células tumorais ou material calcificado. Esta forma caracteriza-se por crescimento rápido, pele avermelhada ou azulada e localização superficial ou até exofitica ${ }^{(13-14)}$, condizendo com o caso deste paciente.

Há descrição na literatura de casos malignos com características de pilomatricoma, sendo bem mais raros que a forma benigna e com diferentes denominações, como: pilomatrix carcinoma, epiteliocarcinoma calcificado de Malherbe, pilomatricoma maligno, carcinoma tricomatrical ou epitelioma calcificado gigante. Considera-se características de malignidade: recorrência após exérese, metástases, infiltrações de tecidos subjacentes, invasão linfática, progressão difusa, presença de necrose ou anormalidades citológicas como desorganização celular, com atividade mitótica atípica e aumentada. O tama-

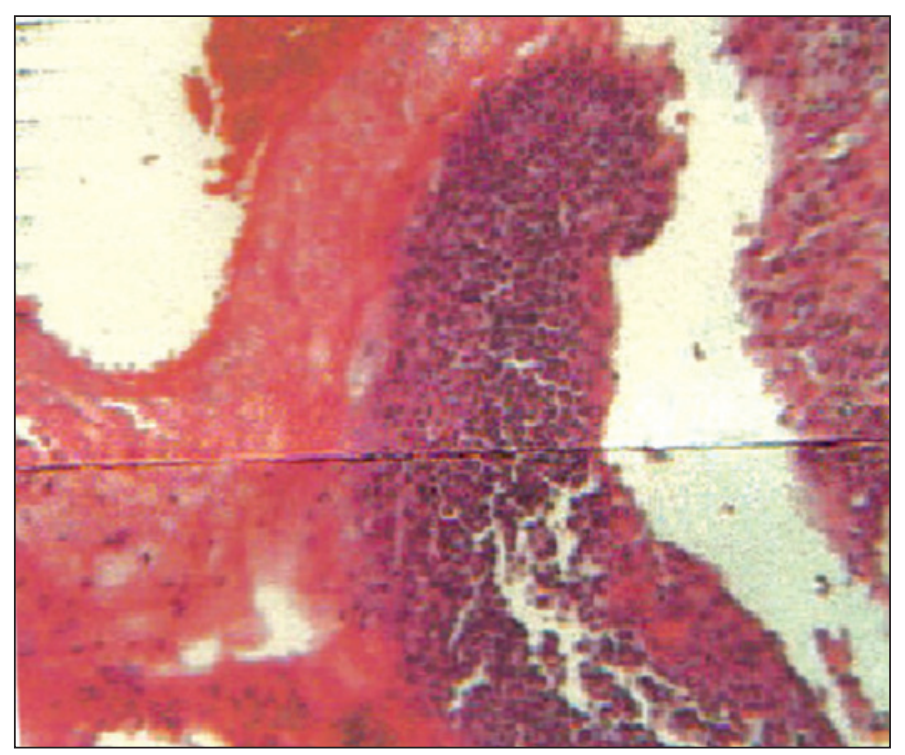

Figura 4 - Foto microscópica de corte da lesão evidenciando: formações císticas com conteúdo amorfo, calcificações, áreas de infiltrado linfoplasmocitário, e presença de áreas distintas contendo células basofílicas e outras contendo células-sombra nho do tumor não determina seu comportamento benigno ou maligno, porém há autores que relacionam maiores tamanhos com potencial aumentado de malignidade ${ }^{(15-16)}$.

Neste caso observamos então algumas características clínicas, inerentes à forma maligna, a qual foi excluída pelo exame anatomopatológico.

O pilomatricoma é um tumor infrequente e de difícil diagnóstico clínico. A recidiva é rara e ainda mais rara é a incidência de formas malignas, no entanto, é fundamental o seguimento do paciente. Este caso ilustra uma situação na qual se poderia suspeitar de forma maligna e exemplifica um incomum caso de recidiva de um tumor já raro.

\section{ABSTRACT}

Pilomatricoma is a benign neoplasm originated in the pilosebaceous follicle wich appears as an intradermal nodule during childhood, in most cases. It is generally covered with normal skin, reaching a diameter of $1.5 \mathrm{~cm}$ on average, and it often shows no recurrence after surgical excision. The authors describe a case in wich a 26-year-old patient presented a tumoral lesion on the upper left superciliary area was submitted to total excision, and diagnosed as pilomatricoma by the histological study. According to the patient, this lesion had a progressive and slow growth of about two years. Eight months after the first surgical intervention, there was a new tumor growth in the region, this time quicker and worsen, with ulceration on the small skin area that covered the lesion, thus leading to malignity suspicion. After the conduction of a new excision within a security margin, the histological test confirmed that it was a pilomatricoma lesion and the piece displayed no sign of malignity. The patient has been followed up for one year, showing no signs of recurrence so far.

Keywords: Pilomatricoma/surgery; Recurrence; Skin neoplasms; Case reports; Human; Male; Adult [Publication type]

\section{REFERÊNCIAS}

1. Pimentel MI, Segura MT, Silva MR. Pilomatricoma. An Bras Dermatol. 1991; 66(1):15-8.

2. Forbis R Jr, Helwig EB. Pilomatrixoma (calcifying epithelioma). Arch Dermatol. $1961 ; 83: 606-18$.

3. Malherbe A, Chenantais J. Note sur l'epitheliome calcifié de glandes sebacées. Bul Soc Anat Paris. 1880;5:169.

4. Sampaio SAP, Castro RM, Rivitti EA. Dermatologia básica. $3^{\underline{a}}$ ed. São Paulo: Artes Médicas; 1985. p.483.

5. Thomas RW, Perkins JA, Ruegemer JL, Munaretto JA. Surgical excision of pilomatrixoma of the head and neck: a retrospective review of 26 cases. Ear Nose Throat J. 1999;78(8):541, 544-6, 548.

6. Cabrera HN, Ferreyra B, Maida, S, Savoia, J. Pilomatrixoma: recopilacion de 25 casos. Rev Argent Dermatol. 1987;68(5):295-8.

7. Moehlenbeck FW. Pilomatrixoma (calcifying epithelioma). Arch Dermatol. 1973;108(4):532-4

8. Carvajal L, Uraga E, Garcia I. Pilomatrixoma. Forma pseudoampollosa y otras variedades clinicas. Med Cut ILA. 1987;15(1):29-34. 
9. Lever WF, Schaumburg-Lever G. Histopathology of the skin. $6^{\text {th }}$ ed. Philadelphia: J.B. Lippincott; 1983. p.530-2.

10. McGavran MH. Ultrastructure of pilomatrixoma (calcifying epithelioma). Cancer. 1965;18(11):1445-56.

11. Pippione M, Depaoli MA, Aloi F. Pilomatricoma. Aspetti clinico-istologici di 100 casi. Giorn Min Dermatol. 1979;114:743-50.

12. Hashimoto K, Nelson RG, Lever WF. Calcifying epithelioma of Malherbe. Histochemical and electron microscopic studies. J Invest Dermatol. 1966;46(4):391-408.
13. Tsoitis G, Mandinaos C, Kanitakis JC. Perforating calcifying epithelioma of Malherbe with a rapid evolution. Dermatologica. 1984;168(5):233-7.

14. Zulaica A, Peteiro C, Quintas C, Pereiro M Jr, Toribio J. Perforating pilomatricoma. J Cutan Pathol. 1988;15(6):409-11.

15. Sasaki CT, Yue A, Enriques R. Giant calcifying epithelioma. Acta Otolaryngol. 1976;102(12):753-5.

16. Wood MG, Parhizgar B, Beerman H. Malignant pilomatricoma. Arch Dermatol. 1984;120(6):770-3.

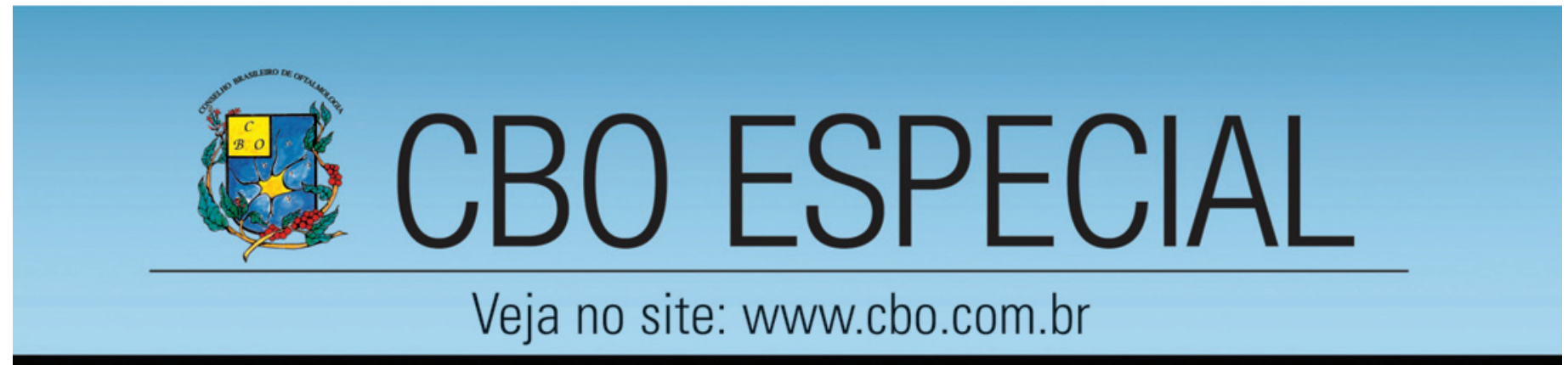

\section{A IMPORTÂNCIA DO EXAME OFTALMOLÓGICO}

